\title{
Design of a New Honeycomb PCF for Ultraflatten Dispersion over Wideband Communication System
}

\author{
Ankita Bapna \\ M.Tech Scholar \\ Department of Electronics and Communication \\ Engineering \\ S.S. College of Engineering, Udaipur, Rajasthan, \\ India
}

\author{
Shivpratap Pandey \\ Assistant Professor \\ Department of Electronics and Communication \\ Engineering \\ S.S. College of Engineering, Udaipur, Rajasthan, \\ India
}

\begin{abstract}
A new kind of honeycomb photonic crystal fiber structure with triangular lattice is proposed. For the proposed design three different air-hole diameters in cladding region is used. To calculate dispersion, 2-D finite difference frequency domain method with the Transparent Boundary conditions (TBC) absorbing boundary conditions is used. Through the numerical simulation and optimizing the geometrical parameters like changing the diameter of air holes (d) for photonic crystal fibers in triangular lattice structure, it has been demonstrated that it is possible to obtain ultra flatten dispersion over a wide wavelength range which lies in second and third telecom window. The ultra flatten dispersion $0 \pm 0.13$ is obtained in the wavelength range 1.4 to $1.71 \mu \mathrm{m}$. The proposed structure is designed using seven ring in which circular air holes are used. The best choice of material for the designing purpose is silica with refractive index 1.458.
\end{abstract}

\section{Keywords}

Effective Refractive Index $\left(\mathrm{n}_{\text {eff }}\right)$, Photonic Crystal Fiber (PCF), Transparent Boundary Condition (TBC).

\section{INTRODUCTION}

As the elegant propagation properties, photonic crystal fibers (PCFs) have engrossed right smart consideration since the first fabrication of PCF in the year of 1996 [1]. Now days several analysis teams are creating constant effort all over the world to determine prevalence of PCFs over conventional fibers due to its novel optical characteristics. It has been reported that PCF will notice as endlessly single-mode guiding fiber [1], manageable nonlinearity [2] and the chromatic dispersion [1]-[8] etc over the large wavelength range.

Several designs for the PCFs have been proposed to achieve nearly zero ultra-flattened chromatic dispersion properties. PCFs with two-defected air hole rings [3], nonlinear PCFs with several kinds of air hole diameters [4], a modified hexagonal PCFs [5] and square PCFs [6] have been proposed. Some of the design procedure becomes complicated to achieve nearly zero ultra-flattened dispersion.

Recently honeycomb [7] PCFs with four kinds of air hole diameters with ultra-flattened dispersion for seven rings was proposed. However, many previous designs are all based on triangular PCFs [1], [3], [5] (see Figure 1), ultra-flattened dispersion properties of square-lattice PCFs [6] (see Figure 2) and a few reports on ultra-flattened dispersion properties of honeycomb PCFs [8], [9], [11]. A few designs were proposed on honeycomb PCF. These designs do not have better dispersion as compare to other design so it is very necessary to investigate ultra-flattened dispersion in honeycomb PCFs.

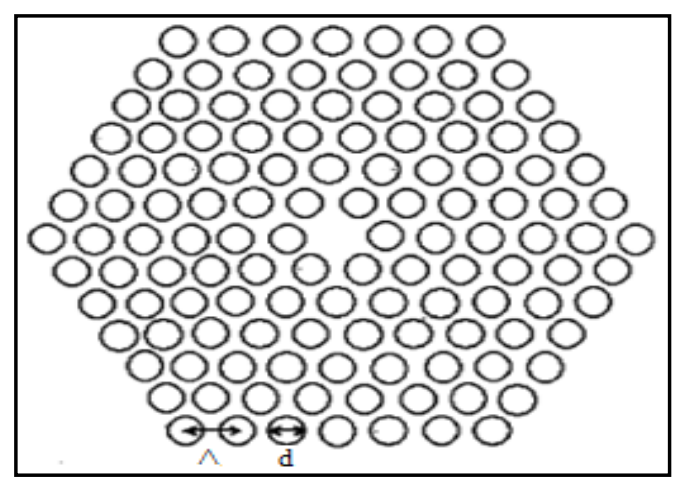

Fig 1: Traditional high index core hexagonal PCF [5]

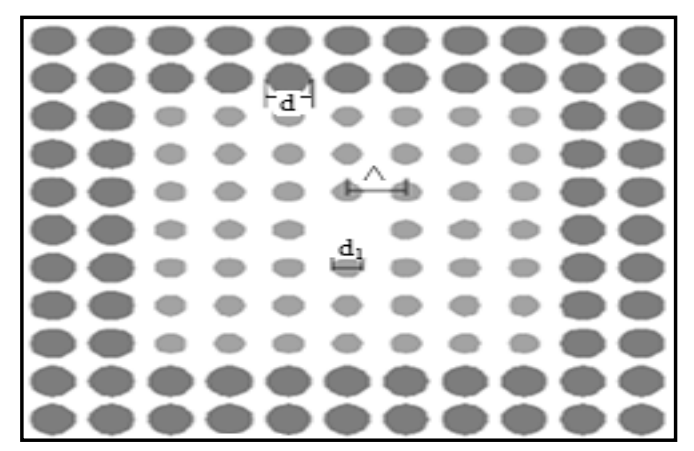

Fig 2: Square lattice PCF with five rings of holes [6]

Now days, exploiting multiple design parameters such as diameter of air holes, shape of the holes (like circular, square, elliptical) and pitch difference, using different kinds of available materials like silica [1]-[5], [7]-[9], $\mathrm{As}_{2} \mathrm{Se}_{3}$, borosilicate crown glass [10], [12] etc., the number of air hole rings and the spacing between these holes facilitates development of PCFs with improved properties.

In this research numerically investigated and proposed a new type of honeycomb Photonic crystal fiber. According to proposed structure, it is possible to design a honeycomb PCFs with nearly zero ultra-flattened chromatic dispersion over second and third optical window by optimizing only three geometrical parameters for air hole diameters as well as a constant pitch $(\wedge)$.

For the designing purpose, used a full vector analysis based method on the finite difference time domain (FDTD) method to analyze the various properties of PCFs. Transparent Boundary conditions (TBC) absorbing boundary are positioned outside the outermost of proposed design. Numerical results shown that the PCFs has nearly zero ultraflattened chromatic dispersion of $0 \pm 0.13 \mathrm{ps} /(\mathrm{nm} . \mathrm{km})$ in all C, 
S, L band which are found to be more flat and wider than those of reported triangular, square and honeycomb PCFs.

\section{ADVANTAGE AND TRANSMISSION WINDOW OF PCFs}

Telecommunications using optical fibers media have become vast. Industries and service providers have to face continuously growing bandwidth demands in all networks areas. However, most of the installed optical fibers are old and exhibit physical characteristics that may limit their ability to transmit high-speed signals in the second and third wavelength window have caused serious concerns using conventional single mode fibers.

The PCF structure works in optical transmission media with zero dispersion and uniform response in different wavelength range. The PCF is having so many unique properties such as high birefringence [1], [6], high nonlinearity [2], [4], wideband dispersion flattened characteristics [1]-[4], [6]-[8], endlessly single mode guiding [1], [2], [6] so that they are widely used in fiber sensors application [1] and fiber lasers as well as supercontinuum generation [10], nonlinear optics [2], [4], telecommunications, soliton [2], medical instrumentations [9] and many more application which are not realizable by conventional optical fiber.

The effects that are contributed to attenuation as well as dispersion depend on the optical wavelength. There are various wavelength bands or windows. In these windows effects are sluggish and these are the maximum positive for transmission. All windows have been regulated and currently characterized bands are the following (see Table 1) [14]

Table 1. Different Optical Windows

\begin{tabular}{|c|c|c|}
\hline Band & Description & $\begin{array}{c}\text { Wavelength } \\
\text { Range }\end{array}$ \\
\hline O band & Original & 1260 to $1360 \mathrm{~nm}$ \\
\hline E band & Extended & 1360 to $1460 \mathrm{~nm}$ \\
\hline S band & Short wavelengths & 1460 to $1530 \mathrm{~nm}$ \\
\hline C band & $\begin{array}{c}\text { Conventional } \\
\text { L band }\end{array}$ & 1530 to $1565 \mathrm{~nm}$ \\
\hline U band & Ultra long Wavelengths & 1625 to $1675 \mathrm{~nm}$ \\
\hline
\end{tabular}

The minimal attenuation occurs at $1.55 \mu \mathrm{m}$ wavelength in silica glass, while the minimum material dispersion reported at $1.312 \mu \mathrm{m}$ wavelength [14]. The preference is occupying on the comparative implication of power loss versus pulse spreading. Suitable light source convenience is also a factor. The first generation optical fiber communication systems performed at the wavelength $870 \mathrm{~nm}$, where the factors, attenuation as well as material dispersion are comparatively high. The more advanced systems basically operate at 1.30 $\mu \mathrm{m}$ and $1.55 \mu \mathrm{m}$ wavelengths [14].

\section{HONEYCOMB STRUCTURE DESIGN AND PCFs SCHEMATIC DIAGRAM}

The schematic cross-section view (see Figure 3) of the proposed PCFs with honeycomb structure is contenting seven rings. The proposed PCFs is composed of circular air holes in the cladding region is arranged in a triangle arrangement, where ' $\Lambda$ ' is the center-to-center spacing between the air holes known as pitch, $d$ is the air hole diameter, $d / \wedge$ is the ratio of normalized diameters of the air holes in the cladding. The background material is silica with refractive index 1.458 and air refractive index 1.0.

In the structure of the proposed honeycomb PCFs design (see Figure 3), some holes are omitted in each ring. In this design, there are three degrees of freedom as inner ring air hole diameter $d_{1}$, outer rings air hole diameter $d_{2}, d_{3}$ and hole to hole spacing is pitch $(\wedge)$. The nearly zero dispersion can be obtained by suitable design of these parameters $d_{1}, d_{2}, d_{3}$ and pitch. The dispersion characteristics are dominantly affected by the air hole sizes of the inner rings as well as pitch. Therefore, after careful selection of the all these parameters, it is possible to achieve desired dispersion properties. The outer rings air hole is kept large enough to improve the chromatic dispersion as well as controlling of confinement loss. In optical communication, dispersion plays an important role as it determines the information carrying capacity fiber. Therefore, it becomes necessary to investigate the dispersion properties of an optical fiber especially for Photonic Crystal Fiber.

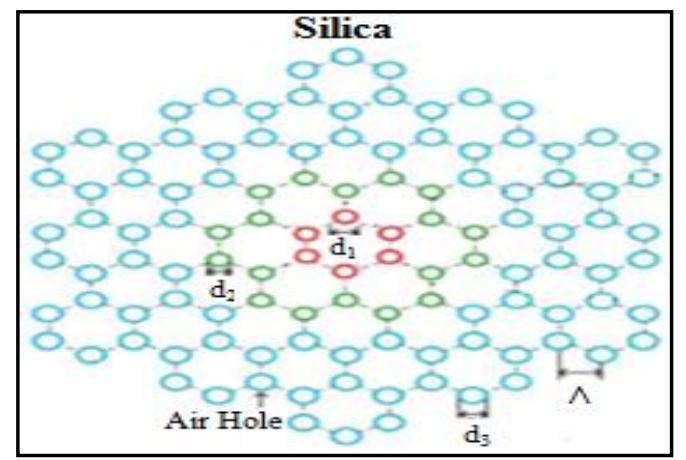

Fig 3: Conventional honeycomb PCFs with seven air hole rings and three different air hole diameter $d$ and pitch $(\wedge)$

Determination of the distortion in signals at the output end is done by dispersion, which is an essential categorization factor of optical fiber. When examination of the Dispersion, gives a suggestion that the distortion to optical signals appear when signal propagate down in the optical fibers in the time communication process is going on. An additional factor which affects the signal is known as delay distortion. For example, creates the expanding in transmitted light pulses so that it will cause restriction of the information carrying capacity of the fiber.

The dispersion (D) is proportional to the subsequent derivative of the $\eta_{\text {eff }}$, with esteem to the wavelength $(\lambda)$ obtained as [1], [2], [4]-[8]:

$$
D=-\left(\frac{\lambda}{c}\right) \frac{d^{2}}{d \lambda^{2}}\left[R_{e}\left(n_{e f f}\right)\right]
$$

Where $\operatorname{Re}\left(\eta_{\text {eff }}\right)$ is the real part of $\eta_{\text {eff }}, \lambda$ is wavelength, and $c$ is the velocity of light in vacuum.

The total dispersion is depends upon the calculation of the sum of the geometrical dispersion (know as waveguide dispersion) and the material dispersion obtained as shown in eq. 2 [1], [5]:

$$
D(\lambda)=D_{g}(\lambda)+\Gamma D_{m}(\lambda)
$$


Where $\Gamma$ is the confinement factor in material, which is close to unity for the most practical PCFs as the modal power is almost confined in the material with high refractive index. $D_{g}(\lambda)$ and $D_{m}(\lambda)$ are waveguide and material dispersion. The waveguide dispersion is depends upon the structure with different air holes diameter as well as pitch but the calculation of material dispersion is done by sellmeier equation as given below [12]

$$
n=\sqrt{1+\frac{\mathrm{A}_{1} \lambda^{2}}{\lambda^{2}-\lambda_{1}^{2}}+\frac{\mathrm{A}_{2} \lambda^{2}}{\lambda^{2}-\lambda_{2}^{2}}+\frac{\mathrm{A}_{3} \lambda^{2}}{\lambda^{2}-\lambda_{3}^{2}}}
$$

Where $\lambda=$ Operating wavelength in $\mu \mathrm{m}$ and the Sellmeier coefficients for Fused silica (fluorine-doped silica 1 mole \%) Sellmeier constants are:

$$
\begin{aligned}
& \mathrm{A}_{1}=0.696166300, \mathrm{~A}_{2}=0.407942600, \mathrm{~A} 3=0.897479400 \\
& \lambda_{1}=4.67914826 \times 10^{-3} \mu \mathrm{m}^{2}, \lambda_{2}=1.35120631 \times 10^{-2} \mu \mathrm{m}^{2}, \\
& \lambda_{3}=97.9340025 \mu \mathrm{m}^{2} .
\end{aligned}
$$

\section{PROPOSED DESIGN SIMULATION AND RESULT}

The proposed honeycomb PCF (see Figure 4) is made up of silica glass and has an array of air holes running along its length.

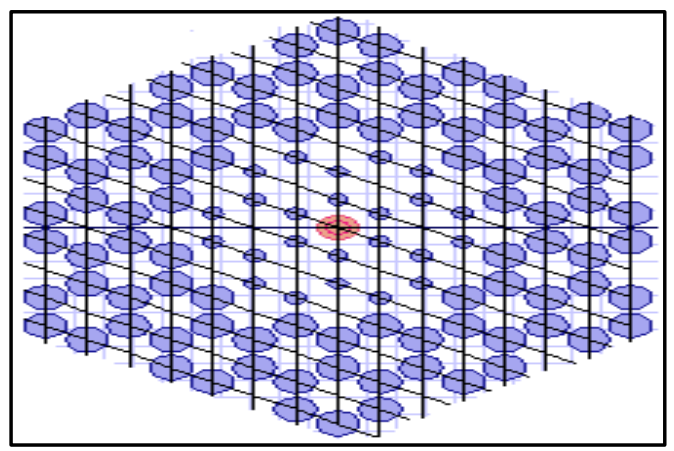

Fig 4: Air-hole distribution of the Proposed honeycomb Structure of Design-5

Now here analysis of the dispersion properties for photonic crystal fiber is to be done. The designed PCF consists of a solid core with a regular array of air holes running along the length of the fiber acting as the cladding.

For the entire configurations analyzed the mean cladding refractive index is lower than the core index. The core material is silica glass which refractive index is 1.458 and the refractive index of cladding air holes is 1 . The lattice structure is in triangular lattice and its structure is honeycomb structure.

Figure 5 shows the effect of changing outer holes diameter $d_{3}$, and other air holes diameter $d_{1}$ and $d_{2}$ is constant as $.67 \mu \mathrm{m}$ and $.705 \mu \mathrm{m}$ on the chromatic dispersion behavior. The outer holes diameter is $1.20 \mu \mathrm{m}, 1.446 \mu \mathrm{m}, 1.60 \mu \mathrm{m}$ for the following design-1, design-2 and design-3. The pitch is 1.8 $\mu \mathrm{m}$ constant for all the designs. Also it can be observed that an increase or decrease of the diameter causes the dispersion parameter to increase or decrease. This has been observed that the dispersion curves are influence of the diameter of holes.

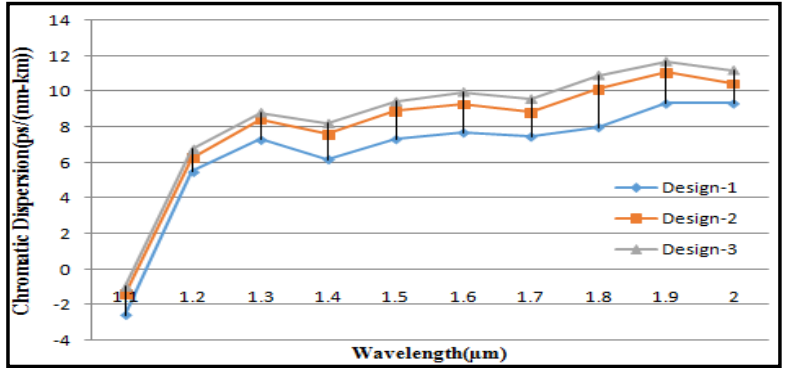

Fig 5: Effective of varying $d_{3}$ diameter while keeping diameter $d_{1}, d_{2}$ and pitch constant

Figure 6 shows the effect of varying the pitch on behavior of chromatic dispersion while keeping the entire diameter constant. The diameters are $\mathrm{d}_{1}=.67 \mu \mathrm{m}, \mathrm{d}_{2}=.705 \mu \mathrm{m}$, $\mathrm{d}_{3}=1.446 \mu \mathrm{m}$ is constant but pitch is varied $1.6 \mu \mathrm{m}, 1.65 \mu \mathrm{m}$, $1.7 \mu \mathrm{m}, 1.85 \mu \mathrm{m}$ from design-4 to design-7.

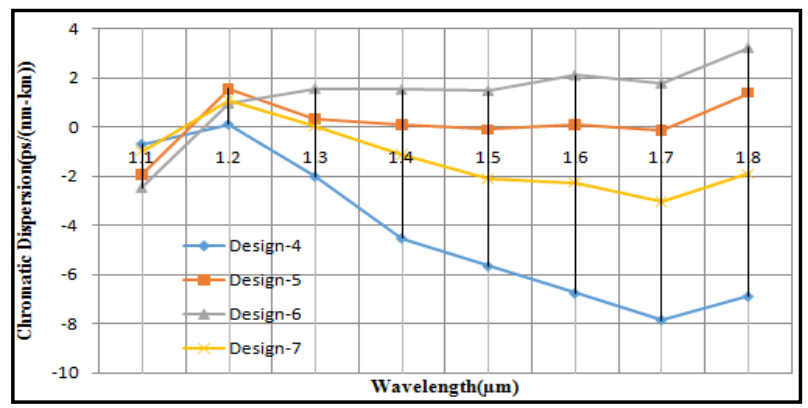

Fig 6: Effective of varying the pitch while keeping diameter $d_{1}, d_{2}$ and $d_{3}$ constant

Also it can be observed that an increase and decrease of pitch causes the dispersion to increase and decrease. The dispersion curves are mainly under the influence of the pitch.

All the result in figures 5 and figure 6 represents the chromatic dispersion as a function of wavelength for the seven rings honeycomb PCFs structure.

In the proposed work there is a comparison between all the seven designs is based on total dispersion (see Figure 5 and Figure 6). For above all the Design-1 to Design-7, it can conclude that Design-5 gives more flatten dispersion for a wide wavelength range as compare to other designs. The typical variation of these design parameters will result in a change of the chromatic dispersion curve nearly zero ultraflattened chromatic dispersion between $\pm 0.13 \mathrm{ps} /(\mathrm{nm} . \mathrm{km})$ for the wavelength range $1.4 \mu \mathrm{m}$ to $1.71 \mu \mathrm{m}$.

Here note that in figure 4 the total air holes area is kept constant as according to the reference paper [7]. Here it can be see that the effect of varying the diameter of all the rings on dispersion behavior while keeping the total air holes area constant.

Table 2. Comparison of various properties of proposed $\mathrm{PCF}$ with reference papers

\begin{tabular}{|c|c|c|c|c|}
\hline \multirow{2}{*}{ PCF } & \multicolumn{4}{|c|}{ Comparison of Model properties } \\
\cline { 2 - 5 } & $\begin{array}{c}\text { Wavelength } \\
\text { Range }\end{array}$ & $\begin{array}{c}\text { Dispersion } \\
\text { ps/ (km-nm) }\end{array}$ & $\begin{array}{c}\text { Flat } \\
\text { Band } \\
\text { (nm) }\end{array}$ & $\begin{array}{c}\mathbf{N r} \\
\mathbf{N}_{\mathbf{\Lambda}} \\
\mathbf{N}_{\mathbf{d}}\end{array}$ \\
\hline Ref. [2] & $\begin{array}{c}1.30 \text { to } 1.60 \\
\mu \mathrm{m}\end{array}$ & $0 \pm 0.6$ & 300 & $5,2,3$ \\
\hline
\end{tabular}




\begin{tabular}{|c|c|c|c|c|}
\hline Ref. [4] & $\begin{array}{c}1.25 \text { to } 1.70 \\
\mu \mathrm{m}\end{array}$ & $0 \pm 1.20$ & 450 & $6,1,3$ \\
\hline Ref. [7] & $\begin{array}{c}1.39 \text { to } 1.70 \\
\mu \mathrm{m}\end{array}$ & $0 \pm 0.2$ & 310 & $7,1,4$ \\
\hline $\begin{array}{c}\text { Proposed } \\
\text { Design-5 }\end{array}$ & $\begin{array}{c}1.4 \text { to } 1.71 \\
\mu \mathrm{m}\end{array}$ & $0 \pm 0.13$ & 310 & $7,1,3$ \\
\hline
\end{tabular}

At the Final, there is a comparison between various properties of the PCFs for telecom and nonlinear optics applications. Therefore, the proposed fiber with a modest number of design parameters, near-zero ultra-flattened dispersion may pave the way for various applications in optics. Table 2 shows the comparison of those fibers taking into flat dispersion, wavelength range and number of design parameters including like number of rings $\left(\mathrm{N}_{\mathrm{r}}\right)$, number of pitch $\left(\mathrm{N}_{\Lambda}\right)$ and number of different diameter of holes $\left(\mathrm{N}_{\mathrm{d}}\right)$ which are used in PCF design respectively.

\section{CONCLUSION}

A new honeycomb index guiding PCFs is numerically investigated ad proposed. The proposed honeycomb PCFs has flattened zero dispersion over a wide wavelength range. In comparison with several other previously research work presented on dispersion-flattened PCFs, the design procedure for this proposed structure would be more efficient and easier because relatively minimized geometrical parameters are required to be optimized and simple for fabrications because of considering less number of air holes. It has been shown that the proposal of honeycomb index-guiding PCFs has nearly zero ultra-flattened chromatic dispersion of $0 \pm 0.13 \mathrm{ps} /(\mathrm{nm} . \mathrm{km})$ in second and third optical window.

As the final conclusion of this research work, the honeycomb PCFs may be suitable for chromatic dispersion management applications as a chromatic dispersion controller, dispersion compensator or as a nonlinear optical system.

\section{REFERENCES}

[1] Jian Liang, Maojin Yun, Weijin Kong, Xin Sun, Wenfei Zhang, Sixing Xi "Highly birefringent photonic crystal fibers with flattened dispersion and low effective mode area" in Optikpage no.2151- 2154, Elsevier GmbH, 2011.

[2] M. Samiul Habib, M. A. Motin, M. I. Hasan, M. Selim Habib, S.M. AbdurRazzak, and M. A. Goffar Khan "Dispersion and Confinement Loss Control with Decagonal Photonic Crystal Fibers for Wideband Transmission Systems" International Conference on Informatics, Electronics \& Vision (ICIEV), IEEE, ISBN978147990400, 2013.

[3] Sejin Lee, Woosung Ha, Hong-gu Choi, Jiyoung Park, Soan Kim and Kyunghwan Oh "Ring Defect Photonic Crystal Fibers for Low loss and Flattened Dispersion" 15th OptoElectronics and Communications Conference (OECC2010) Technical Digest, July 2010, Sapporo Convention Center, Japan.

[4] Md. Sharafat Ali, Aminul Islam, Redwan Ahmad, A. H. Siddique, K M Nasim, M A G Khan, M Samiul Habib "Design of Hybrid Photonic Crystal Fibers for Tailoring Dispersion and Confinement Loss" International
Conference on Electrical Information and Communication Technology (EICT), IEEE, ISBN978147992299, 2013.

[5] JingyuanWanga, Chun Jiangb, Weisheng Hub, MingyiGaob, HongliangRenb "Dispersion and polarization properties of elliptical air-hole-containing photonic crystal fibers" Optics \& Laser Technology, Elsevier Ltd. 2007.

[6] TAN Xiao-ling, GENG You-fu, TIAN Zhen, WANG Peng and YAO Jian-quan "Study of ultra flattened dispersion square-lattice photonic crystal fiber with low confinement loss" Optoelectronics Letters, Vol.5 No.2, 1 March 2009.

[7] Nghiem Xuan Tam, Nguyen Xuan Son, Pham Hong Thai Nguyen Hoang Haia "New Proposal of Chromatic Dispersion Control in Honey Comb Photonic Crystal Fiber for Broadband Communication" Fifth International Conference on Communications and Electronics (ICCE), 2014, IEEE, ISBN-9781479950492, July 30 2014-Aug. 12014

[8] NihalAreed "Ultra-Flattened Dispersion Honeycomb Lattice Photonic Crystal Fiber" 5th International Conference: Sciences of Electronic, Technologies of Information and Telecommunications, March 22-26, 2009.

[9] S.S. Mishra and Vinod Kumar Singh "Comparative Study of Fundamental Properties of Honey Comb Photonic Crystal Fiber at $1.55 \mu \mathrm{m}$ Wavelength" Journal of Microwaves, Optoelectronics and Electromagnetic Applications, Vol. 10, No. 2, December 2011.

[10] R. Buczynski, B. Siwicki, M. Klimczak, D. Pysz, T. Martynkien, P. Skibinski, C. Radzewicz, and R. Stępień, "Supercontinuum Generation in All Solid Photonic Crystal Fiber with Flat All-Normal Dispersion" $18^{\text {th }}$ Microoptics Conference (MOC'13), Tokyo, Japan, Oct. 27 - 30, 2013

[11] Sh. Mohammad nejad, M. Aliramezani, M. Pourmahyabadi "Novel Design of an Octagonal Photonic Crystal Fiber with Ultra-Flattened Dispersion and Ultra-Low Loss" IEEE third International Conference on Broadband Communications, Information Technology \& Biomedical, Applications, ISBN- 978076953453, 2008.

[12] Shumaila Akbar, Dr. Navneet Agarwal, Dr. Suriti Gupta "Elliptical hybrid cladding Borosilicate photonic crystal fiber Design for minimum chromatic dispersion" $11^{\text {th }}$ International Conference on Wireless and Optical Communications Networks (WOCN), IEEE, ISBN978147993156, 2014.

[13] Juan Juan $\mathrm{Hu}$, Ping Shum, Chao Lu and Guobin Ren "Generalized Finite-Difference Time-Domain Method Utilizing Auxiliary Differential Equations for the FullVectorial Analysis of Photonic Crystal Fibers" IEEE Photonics Technology Letters, Vol. 19, No. 24,December 15, 2007.

[14] Bahaa E. A. Saleh, Malvin Carl Teich "Fundamental of photonics" ISBN- 978-0-471-35832-9 John Wiley \& Sons, Inc Publication, 2007. 